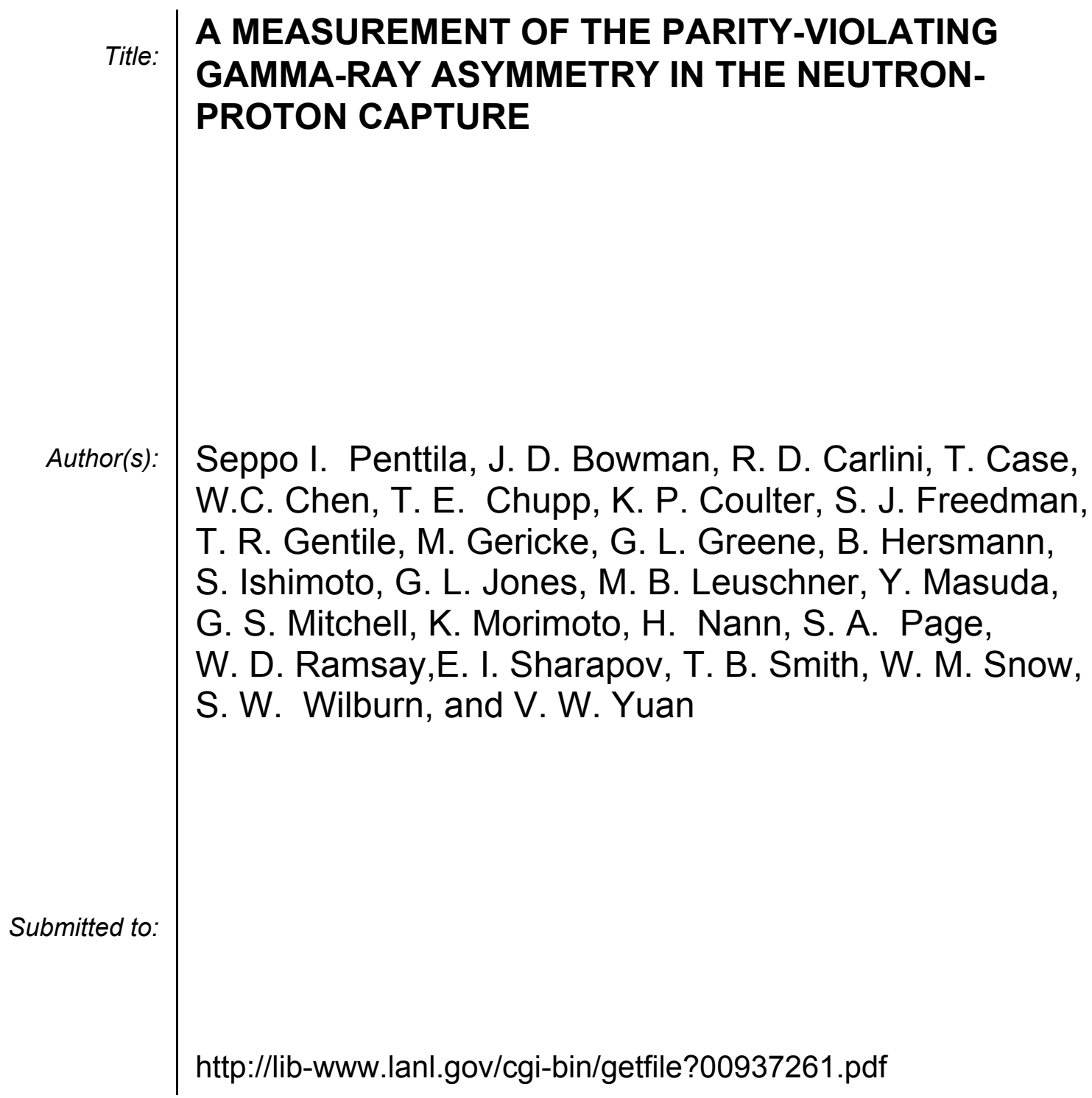




\title{
A MEASUREMENT OF THE PARITY-VIOLATING GAMMA-RAY ASYMMETRY IN THE NEUTRON-PROTON CAPTURE
}

\author{
THE NPDGAMMA COLLABORATION

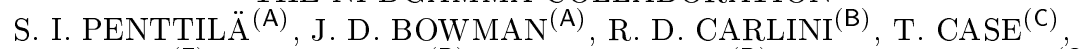

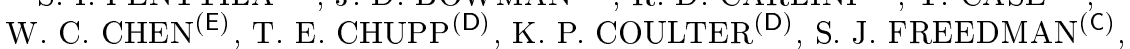

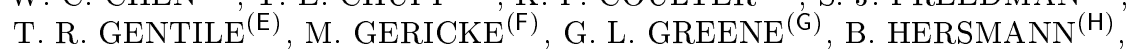

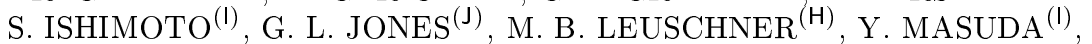

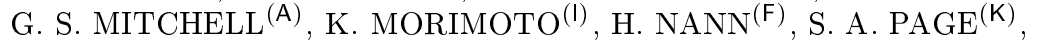 \\ W. D. RAMSAY (M) , E. I. SHARAPOV $(\mathrm{N})$, T. B. $\operatorname{SMITH}^{(\mathrm{O})}$, W. M. $\operatorname{SNOW}^{(\mathrm{F})}$, \\ S. W. WILBURN ${ }^{(A)}, \operatorname{AND~Y.~W.~YUAN~}^{(A)}$ \\ ${ }^{a}$ Los Alamos National Laboratory, Los Alamos, NM 87545, USA \\ ${ }^{b}$ Thomas Jefferson National Accelerator Facility, Newport News, VA 23606 , \\ USA \\ ${ }^{c}$ University of California, Berkeley, CA 94720, USA \\ ${ }^{d}$ University of Michigan, Ann Arbor, MI 48109, USA \\ ${ }^{e}$ National Institute of Standards and Technology, Gaithersburg, MD 20899, USA \\ ${ }^{f}$ Indiana University, Bloomington, IN 47405, USA \\ ${ }^{g}$ University of Tennessee, TN 37996, USA \\ ${ }^{h}$ University of New Hampshire, Durham, NH 03824, USA \\ ${ }^{i}$ KEK National Laboratory, Tsukuba, Japan \\ ${ }^{j}$ Hamilton College, Clinton, NY 13323, USA \\ ${ }^{k}$ University of Manitoba, Winnipeg, MB R3T2N2, Canada \\ ${ }^{m}$ TRIUMF, Vancouver, BC V6T2A3, Canada \\ ${ }^{n}$ Joint Institute for Nuclear Research, Dubna, Russia \\ ${ }^{\circ}$ University of Dayton, Dayton, $\mathrm{OH}$ 45469-1679, USA
}

The $\vec{n}+p \rightarrow d+\gamma$ experiment under construction at LANSCE studies the weak interaction between neutrons and protons. The experiment will measure the directional dependence of the parity-violating $\gamma$-ray asymmetry, $A_{\gamma}$, in the polarized cold neutron capture by para-hydrogen. The goal is to measure $A_{\gamma}$ with uncertainty of $0.5 \times 10^{-8}, 10 \%$ of its predicted value. $A_{\gamma}$ primarily isolates the $\Delta I=1$ component of the hadronic weak interaction, and thus will determine the long-range weak pion-nucleon coupling constant $H_{\pi}^{1}$. The experiment is carefully designed for the LANSCE pulsed spallation neutron source to achieve the proposed statistical precision and to control systematic errors. We discuss the experiment and its status. 


\section{Introduction}

Nucleons are bound together to form nuclei by the strong interaction. Nucleons and nuclei decay through the relatively feeble weak interaction. The existence of the weak decays of nucleons implies that pairs of nucleons have to interact weakly as well as strongly. Experimentally, it is confirmed that the electromagnetic and strong interactions are parity conserving, whereas the weak interaction is parity violating $(P V)$. A signature of the weak interaction is thus a $P V$ asymmetry. This signature is used to experimentally isolate small effects of the weak interaction in the presence of the much $\left(10^{6}-10^{7}\right.$ times) stronger strong interaction. Although many $P V$ effects have been observed in nuclei, a quantitative description of these results has yet to be developed.

The standard model explains well how point-like leptons and quarks interact weakly by exchanging weak bosons, $\mathrm{W}^{+}, \mathrm{W}^{-}$, and $\mathrm{Z}^{0}$. After more than a decade of experimental and theoretical effort, we still have only a phenomenological description for the weak force between hadrons. Experiments and calculations are difficult because of the strong interaction that modifies the weak couplings between the quarks and the weak bosons. In principle, these modifications can be calculated in the standard model. However, at present the strong interaction can be treated only at a very large momentum transfer in a framework of perturbative QCD. In order to describe the weak interactions of nucleons, the weak couplings must be determined experimentally. Highly sensitive experimental approaches are required to observe tiny $P V$ effects that are hidden behind the strong interaction physics.

At low energies the weak interaction between hadrons can be considered as a point interaction of two currents - the charged and neutral currents. From the isospin structure of the non-leptonic strangeness conserving $(\Delta S=0)$ part of the hadronic weak interaction, we obtain isospin components $\Delta I=0$ and 2 for the weak charged current and $\Delta I=0,1$, and 2 for the neutral current ${ }^{1,2}$. Therefore, it can be assumed that the neutral current should dominate the isospin $\Delta I=1$ (isovector) channel of the hadronic weak interaction. The $\gamma$-asymmetry in $\vec{n}+p \rightarrow d+\gamma$ is a measure of the $\Delta S=0$ and $\Delta I=1$ part of the hadronic weak interaction - the neutral current part. Contributions of the neutral current to hadronic reactions have not yet been experimentally observed at low energies.

The range of the $N N$ interaction is $1 / m_{\pi} \approx 1.5 \mathrm{fm}$. On the other hand, the Compton wavelengths of the heavy weak bosons are $1 / M_{W} \approx 10^{-3}$ $\mathrm{fm}$. Thus, the hadronic weak interaction cannot be explained as a simple 
exchange of weak bosons between nucleons, a more complicated model is needed to describe how the long-range weak force is communicated between nucleons.

Conventionally, at low energies the weak interaction between nucleons is described as the one-meson exchange model where one of the vertices is $P V$ and the other is parity conserving. The model requires six weak coupling constants, $H_{m}^{\Delta I}$, to describe the weak nuclear potential. Here $\Delta I$ gives the isospin transfer and $m$ the effective meson exchanged $\left(\pi^{ \pm}\right.$, $\rho$, or $\omega)^{3}$. A number of experiments have attempted to measure these weak couplings from a variety of observables ${ }^{2,4,5}$. However, only the $P V$ longitudinal asymmetry results in $p p$ scattering, $A_{L}^{p p}$, are sensitive enough to observe $P V$ effects ${ }^{6}$. $A_{L}^{p p}$ depends upon a combination of short-range $\rho$ and $\omega$ couplings, and additional experiments are required to constrain the individual couplings. The most important among the weak couplings is the weak pion-nucleon coupling, $H_{\pi}^{1}$ a, which represents the long-range part of the weak interaction as does the strong pion-nucleon coupling for the strong interaction.

Several experiments including a prevoius $\vec{n}+p \rightarrow d+\gamma$ experiment ${ }^{7}$, have tried to isolate $H_{\pi}^{1}$. The most significant results have been obtained from the five internally consistent measurements of the circular polarization $P_{\gamma}$ of a $\Delta I=1 P V$ gamma-ray transition in ${ }^{18} \mathrm{~F}^{8}$. The combined result, shown in figure 1 as a vertical band, gives an upper limit for $H_{\pi}^{1}$ that is considerably smaller than predicted by the explicit SU(6)-quark model analysis ${ }^{3}$ - DDH and its reasonable range in figure 1.

Our knowledge of $H_{\pi}^{1}$ was thrown into doubt by a measurement of the nuclear spin-dependent $P V$ effect in the ${ }^{6} S-{ }^{7} S$ transitions in the ${ }^{133} \mathrm{Cs}$ atom ${ }^{9}$. The result of this very high-precision atomic $P V$ measurement allowed for the first time the extraction of a value for the cesium static nuclear anapole moment in the ground state ${ }^{10}$. Recently, a full 2-body calculation of a cesium anapole moment has been used to place constraints on $H_{\pi}^{1}$ and the short-range, $\Delta I=0 \rho$-nucleon coupling, $H_{\rho}^{011}$ (see figure 1 ). The cesium result implies a central value for $H_{\pi}^{1}$ two times larger than the $\mathrm{DDH}^{12}$. $H_{\pi}^{1}$ deduced from measurements of $P V$ longitudinal asymmetries in neutron resonances ${ }^{5}$ also supports a possible large value of $H_{\pi}^{1}{ }^{13}$.

The constraint for the weak couplings given by atomic $P V$ experiments on ${ }^{205} \mathrm{Tl}{ }^{14}$ is shown in figure 1 . These measurements were not sensitive enough to detect the anapole moment contribution and thus give only in-

${ }^{\mathrm{a}}$ We have used the notation $H_{\pi}^{1}=F_{\pi}=g_{\pi} f_{\pi} / \sqrt{32}$ and $H_{\rho}^{0}=F_{0}=-g_{\rho} h_{\rho}^{0} / 2$ where $g_{\pi}$ and $g_{\rho}$ are the strong coupling constants. 


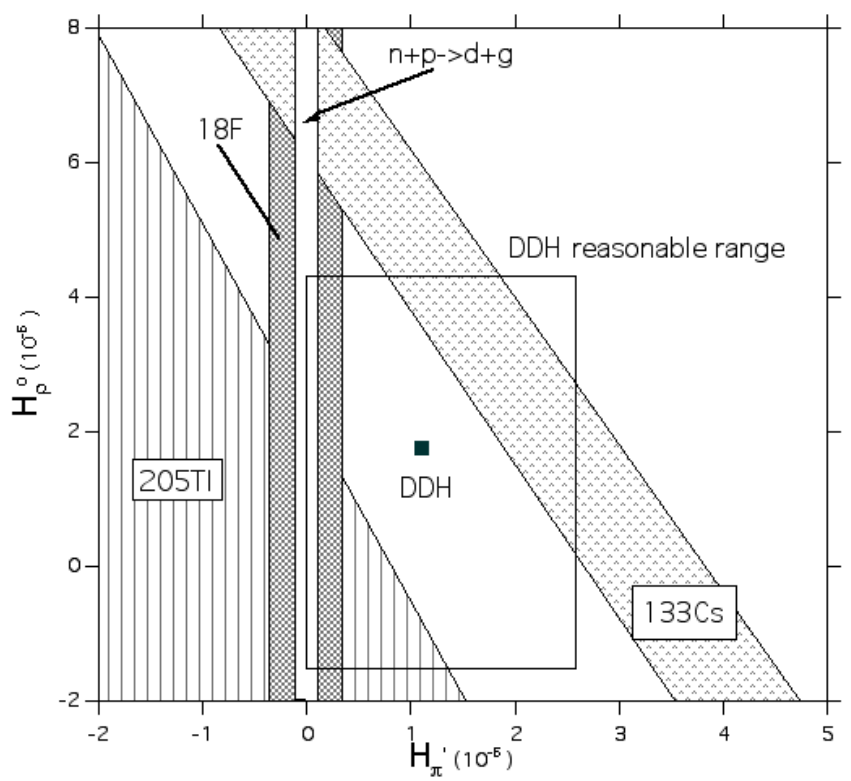

Figure 1. Constraints on the weak coupling constants $H_{\pi}^{1}$ and $H_{\rho}^{0}$ extracted from the ${ }^{18} \mathrm{~F},{ }^{133} \mathrm{Cs}$, and ${ }^{205} \mathrm{Tl}$ experiments. Also shown are the DDH prediction, its reasonable range, and the goal uncertainty of the $\vec{n}+p \rightarrow d+\gamma$ experiment.

teresting limits to the hadronic weak interaction ${ }^{10,11}$. It is evident that the constraints shown in figure 1 have no single solution. To resolve the controversy between the ${ }^{18} \mathrm{~F}$ and ${ }^{133} \mathrm{Cs}$ results and between different theoretical predictions ${ }^{15}$, a new high precision experiment to measure $A_{\gamma}$ in $\vec{n}+p \rightarrow d+\gamma$ is needed. Since the $n p$ capture involves only a 2 -body system, the result will give a strong constraint on the $P V N N$ interaction that is free from nuclear theory uncertainties present in the interpretation of the results from complex nuclei such as cesium and fluorine.

Due to the low center-of-mass energy, the $n p$ capture mainly takes place in a $s$-wave, either in a singlet initial scattering state, ${ }^{1} S_{0}$, or in a triplet state, ${ }^{3} S_{1}$. The ground state of the deuteron is ${ }^{3} S_{1}$, (ignoring $4 \%$ tensor part).

There is merely one regular magnetic dipole $M 1$ transition from the initial scattering state ${ }^{1} S_{0}$ into the deuteron, because the ${ }^{3} S_{1}$ state should be orthogonal to the deuteron bound state. Due to the weak forces $P$ states are mixed to the initial and the final states which are excited by the isovector $\Delta I=1$ and isoscalar $\Delta I=0$ transition operators. These admixtures produce irregular electric dipole $E 1$ transitions. A $M 1-E 1$ 
interference leads to the directional gamma-ray asymmetry $A_{\gamma}$.

The goal of the $\vec{n}+p \rightarrow d+\gamma$ experiment at LANSCE is to measure $A_{\gamma}$ with an uncertainty of $0.5 \times 10^{-8}$ and thereby unambiguously determine $H_{\pi}^{1}$, since $A_{\gamma} \sim-0.045\left(H_{\pi}^{1}-0.02 H_{\rho}^{1}+0.02 H_{\omega}^{1}+0.04 H_{\rho}^{\prime 1}\right)$. The $\vec{n}+p \rightarrow d+\gamma$ experiment has been carefully designed for the LANSCE pulsed spallation neutron source to achieve the statistical limit and to keep systematic errors an order of magnitude smaller than the statistical uncertainty.

\section{The $\vec{n}+p \rightarrow d+\gamma$ experiment}

A new class of precision fundamental physics experiments can be designed for pulsed spallation neutron sources to take full advantage of the high neutron flux and knowledge of the neutron energy through the time-offlight (tof) measurement. The neutron energy information can be then used to control systematic errors in situ. A good example of this kind of experiment is the $\vec{n}+p \rightarrow d+\gamma$ experiment that is under construction at LANSCE by the NPDGamma Collaboration. The setup of the experiment is shown in figure 2 .

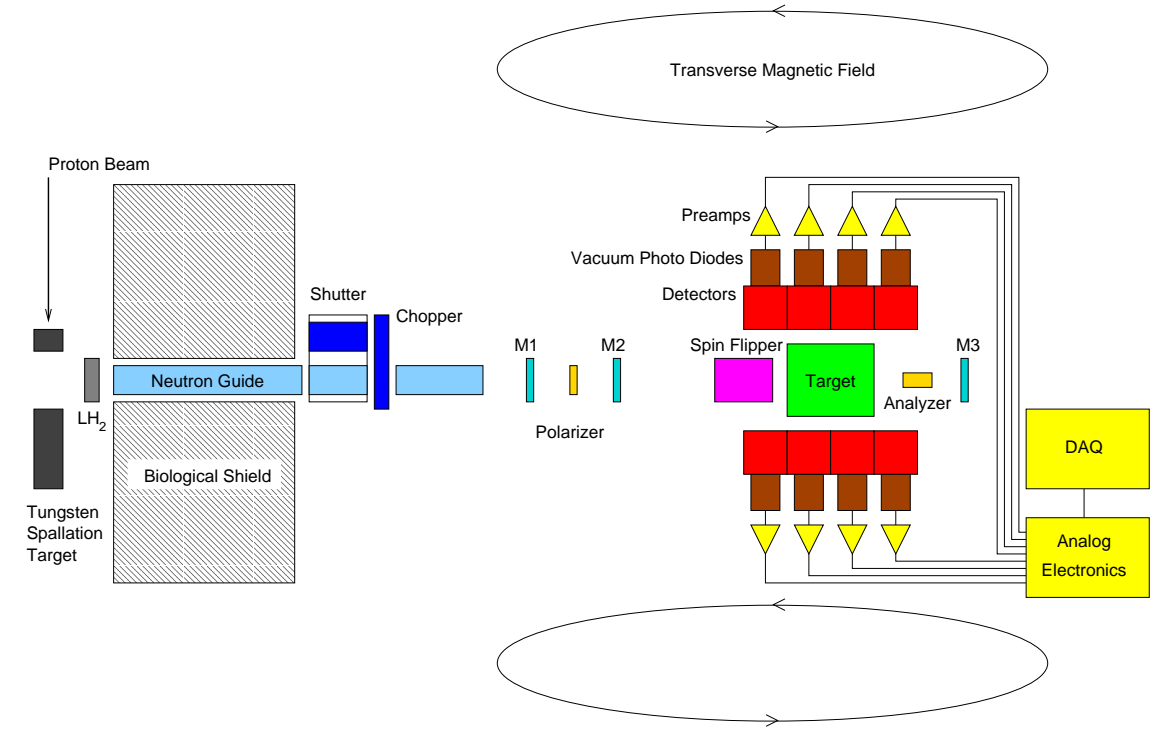

Figure 2. Diagram of the $\vec{n}+p \rightarrow d+\gamma$ experiment, showing the major components. 


\subsection{Pulsed cold neutron flux from the LANSCE pulsed spallation source}

Flight path 12 at the Manuel Lujan Jr. Neutron Center views the upper tier cold $\mathrm{H}_{2}$ moderator of the LANSCE $20-\mathrm{Hz}$ spallation source. The details of the source and the predicted moderator brightness are discussed in Ref. ${ }^{16}$. The moderated neutrons are guided to the experiment, located at $22 \mathrm{~m}$ from the source, by a $9.5 \times 9.5 \mathrm{~cm}^{2}$ neutron guide with the reflectivity of $\Theta_{c}=3.5$ times that of nat. Ni. A frame defination chopper is mounted $9.5 \mathrm{~m}$ from the moderator. With its two independent rotors an arbitrary tof frame width can be selected. The calculated neutron flux as a function of tof at the end of the $20 \mathrm{~m}$ guide is shown in figure 3 .

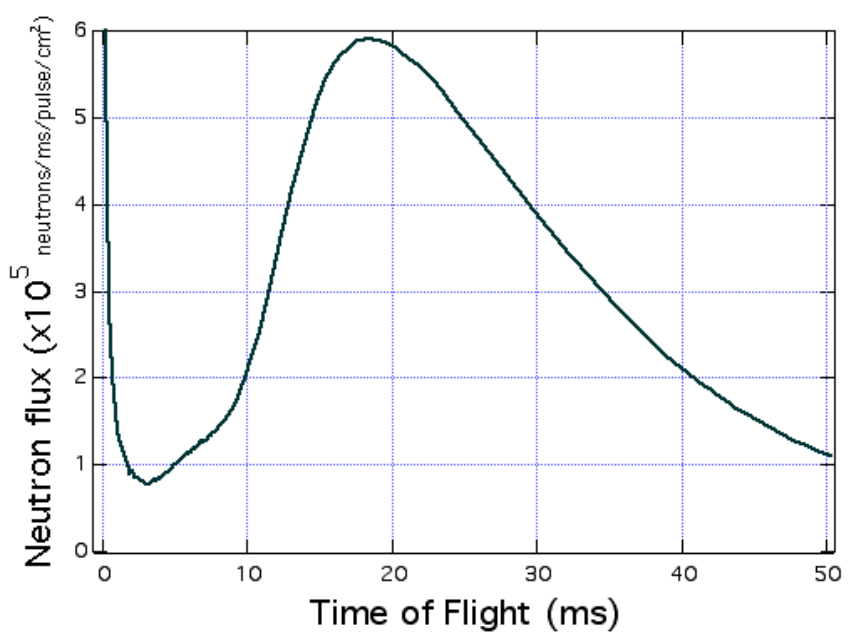

Figure 3. Calculated neutron flux of flight path 12 as a function of time-of-flight at the end of a $20-\mathrm{m}$ long neutron guide with an average proton current of $150 \mu \mathrm{A}$.

\section{2. ${ }^{3} \overrightarrow{H e}$ neutron spin filter}

The neutrons will be polarized by passing them through optically-polarized ${ }^{3} \mathrm{He}$ gas ${ }^{17,18}$. Because of the large spin dependent $n-{ }^{3} \mathrm{He}$ capture cross section, the neutrons with opposite (parallel) spin direction to the ${ }^{3} \mathrm{He}$ polarization will be absorbed (transmitted). After the spin filter the neutron polarization is $P_{\mathrm{n}}=\tanh \left[n l \sigma_{p}\left(E_{\mathrm{n}}\right) P_{\mathrm{H} e}\right]$ where $n$ is the number density of the ${ }^{3} \mathrm{He}$ gas, $l$ is the length of the gas, $\sigma_{p}\left(E_{\mathrm{n}}\right)$ is the $n-{ }^{3} \mathrm{He}$ polarized cross section ${ }^{18}$, and $P_{\mathrm{He}}$ is ${ }^{3} \mathrm{He}$ polarization. To cover the full beam, we have 
constructed ${ }^{3} \mathrm{He}$ cells with a diameter of up to $11 \mathrm{~cm}$. For optimum performance the ${ }^{3} \mathrm{He}$ thickness of $1.2 \times 10^{20}{ }^{3} \mathrm{He} / \mathrm{cm}^{2}$ is required. ${ }^{3} \mathrm{He}$ polarization of $52 \pm 3 \%$ has been demonstrated with these cells. The use of the ${ }^{3} \mathrm{He}$ spin filter is one of the key features of the $\vec{n}+p \rightarrow d+\gamma$ experiment. The ${ }^{3} \mathrm{He}$ spin filter has a number of advantages compared to supermirror polarizers. Its acceptance angle is large and a neutron capture on ${ }^{3} \mathrm{He}$ does not create $\gamma$-rays. The spin filter can be operated in a magnetic field of $10 \mathrm{G}$ with $\Delta B$ less than $1 \mathrm{mG} / \mathrm{cm}$. The neutron spin polarization can be reversed without changing the direction of the 10-G guide field by adiabatic fast passage of the ${ }^{3} \mathrm{He}$ spin. This allows the design of very compact experimental setups.

A thick polarized ${ }^{3} \mathrm{He}$ cell is mounted behind the apparatus to analyze polarization of the transmitted beam.

\subsection{Neutron beam monitors}

The neutron flux at the end of the guide is monitored by a thin (1\% loss in the gas and $\sim 2 \%$ losses in the $\mathrm{Al}$ windows) ${ }^{3} \mathrm{He}$ ionization chamber (M1 in figure 2). The monitor is filled with a gas mix consisting of ${ }^{3} \mathrm{He}$, ${ }^{4} \mathrm{He}$, and $\mathrm{N}_{2}$ to one standard atmosphere. The efficiency is controlled by adjusting the partial pressure of ${ }^{3} \mathrm{He}$. M1 and the second identical ${ }^{3} \mathrm{He}$ monitor (M2), after the spin filter, are used to monitor the performance of the polarizer through a transmission measurement. The transmission of an unpolarized neutron beam through a polarized ${ }^{3} \mathrm{He}$ cell is given by $T_{\mathrm{n}}=T_{\mathrm{n}}^{0} \cosh \left[n l \sigma_{p}\left(E_{\mathrm{n}}\right) P_{\mathrm{He} e}\right]$. The third monitor, similar but thicker ${ }^{3} \mathrm{He}$ ion chamber (M3) after the apparatus, measures the fraction of the beam that will pass through the $\mathrm{LH}_{2}$ target. Together with M2 and M3 the para-ortho-concentration in the target is monitored.

\subsection{Neutron spin reversal with a RF spin flipper}

Neutron spin reversal is the main control of the systematic errors of the experiment. The neutron spin can be reversed slowly either by reversing the polarization direction of the ${ }^{3} \mathrm{He}$ spin filter or by changing the polarity of the guide magnetic field. A fast neutron spin flip is accomplished by a RF spin flipper (RFSF). The RFSF is based on neutron spin resonance; an RF field $\vec{B}_{1} \cos (\omega t)$ is along the beam axis, perpendicular to the homogeneous vertical $(\mathrm{d} B / B<1 \mathrm{mG} / \mathrm{cm})$ magnetic field of $B_{0}=10 \mathrm{G}$. The combination of the two fields adiabatically rotates the neutron spin. At resonance, $\omega=\omega_{0}$ ( $\omega_{0}$ is the Larmor frequency), the rotation angle $\Theta$ of the neutron spin when passing through the $30 \mathrm{~cm}$ long spin flipper depends upon the amplitude $B_{1}$ and the time $\Delta t$ that a neutron spends in the $30-\mathrm{kHz} \mathrm{RF}$ 
field of the flipper, where $\Theta=\gamma B_{1} \Delta t$. Here $\gamma$ is the gyromagnetic ratio of neutron spin. The neutrons with different energies enter the RFSF at different times and, therefore, the amplitude $B_{1}$ has to be tuned to match the entering neutron energy. For a spin reversal in a large neutron energy range $B_{1}(t)=(\pi L / \gamma l)(1 /$ tof $)$. Here $L=22 \mathrm{~m}$ is the length of the flight path and $l$ is the length of the spin flipper. The spin-flip efficiency of the RFSF is measured to be better than $97 \%$ over the beam volume ${ }^{19}$. The $30 \mathrm{~cm}$ length of the flipper satisfies the adiabaticity requirement of the spin reversal for neutron energies that are the interest of the $\vec{n}+p \rightarrow d+\gamma$ experiment. Because of the resonance requirement, the spin reversal efficiency of the RFSF is sensitive to small changes in $B_{0}$. The RFSF does not change the kinetic energy of the neutron and so the phase space of the beam in the flipped and unflipped states is identical. The RFSF has to be able to flip the neutron spin at a frequency of $20 \mathrm{~Hz}$.

Resonance RF spin flippers based on the use of magnetic gradients cannot be used in this experiment because they could create the Stern-Gerlach force $\vec{\mu} \cdot \Delta \vec{B}$ and lead to a false asymmetry.

\subsection{Liquid para-hydrogen target}

Most of the neutrons are captured in a 20-1 liquid para-hydrogen target. The $n p$ scattering cross section in ortho-hydrogen is larger than in parahydrogen so a high concentration of the para-hydrogen is a basic requirement for the target. Scattering from ortho-hydrogen will depolarize the neutrons and thus dilute the $P V$ asymmetry; also most of the neutrons would be scattered out from the target before capture. The target will be run at $17 \mathrm{~K}$ to keep the ortho-hydrogen concentration less than $0.05 \%$. For neutrons with energy greater than $15 \mathrm{meV}$, depolarization will occur in liquid para-hydrogen by spin-flip scattering and, therefore, no $P V$ effect can occur. Neutrons with energies less than $15 \mathrm{MeV}$ do not have enough energy to depolarize by flipping the proton spin in the para-hydrogen and will, therefore, show a $P V$ effect. These two neutron energy regions can be separated in DAQ per neutron pulse; when the neutron energy is higher than $15 \mathrm{meV}$, the collected data should provide an in situ null result. Although the target has a length of $20 \mathrm{~cm}$ and a diameter of $20 \mathrm{~cm}$, only $65 \%$ of the beam will be captured; the rest of the beam will scattered out of the target or pass through the target. The neutrons scattered out of the target have to be absorbed before they activate the CsI detectors or create any unwanted false asymmetries when interacting with materials in the apparatus. The fraction of the neutrons that pass the target is used to monitor the ortho-para ratio in the target. 


\subsection{A detector for 2.2-MeV gamma rays}

The $2.225-\mathrm{MeV} \gamma$-rays from the $n p$ capture reaction are detected by an array of $48 \mathrm{CsI}(\mathrm{Tl})$ crystals that are arranged around the $\mathrm{LH}_{2}$ target to cover about $3 \pi$ solid angle. CsI was selected because the interaction length of the $2.2 \mathrm{MeV} \gamma$ 's is about $5 \mathrm{~cm}$, so a $15 \times 15 \times 15 \mathrm{~cm}^{3}$ CsI crystal stops about $95 \%$ of $\gamma$ rays. In addition, $\mathrm{CsI}(\mathrm{Tl})$ produces a large number of photoelectrons; over 1000 photoelectrons per $\mathrm{MeV}$ have been measured for these crystals with vacuum photo diodes. And $\mathrm{CsI}(\mathrm{Tl})$ has been found to be rather radiation-hard up to doses of more than $50 \mathrm{krad}^{20}$.

The peak $\gamma$ rate in each detector crystal will be over $1 \times 10^{8} \gamma$ per second, which is too high for single-pulse counting. Therefore, current mode signal processing will be used. Light from each of the crystals is converted to current by an 8 -cm diameter S20 alkali photocathode of a vacuum photo diode (VPD) ${ }^{21}$. VPDs are highly linear $\left(<10^{-4}\right)$, and they are insensitive to magnetic fields (gain change of $\Delta G / \Delta B<10^{-4} / \mathrm{G}$ in a $10-\mathrm{G}$ field). The gain of a VPD is one, therefore low-noise and high-gain current-tovoltage preamplifiers were developed to amplify small photocathode currents. The signals from the upper and lower hemispheres of the detector will be summed and recorded by transient digitizers over a selected, about 30 ms wide, tof frame. The VME/Linux based DAQ system can read 1000 samples per neutron pulse, but data reduction is needed. Signals will be averaged so that only 50 data values per neutron pulse per 48 detectors will be stored. Because of the up-down symmetry, the detector has to be affectively aligned to within $\pm 20 \mathrm{mrad}$ with respect to the guide field $\vec{B}_{0}$. This will prevent a small parity-allowed left-right asymmetry ${ }^{22}$ from leaking into the up-down symmetry and cause a false $P V$ signal.

\section{Statistic and systematical errors}

The statistical fluctuations in the signal, counting statistics, will appear as a shot noise in the photocathode current and can be estimated to be $I_{\mathrm{sn}}=\sqrt{2 q I} \approx n_{\mathrm{pe}} e \sqrt{2 \times \text { rate }}$, where $q$ is the deposited charge per $2.2-\mathrm{MeV}$ $\gamma$ and $I$ the vacuum photo current, $n_{\mathrm{pe}}$ is number of photoelectrons, $e$ is the charge of the electron, and rate is the $\gamma$ rate. Using the numbers given above, we obtain $I_{\mathrm{sn}} \approx 6 \mathrm{pA} / \sqrt{\mathrm{Hz}}$. This shot noise defines the number of neutron macropulses needed to achieve the uncertainty of $0.5 \times 10^{-8}$. The effective run time estimate is about 10 months with the average proton current of $150 \mu \mathrm{A}$.

Electrical noise of the detector system has to be kept significantly smaller than the counting statistics in order for current mode detection 
to work. The amplifier noise was measured to be $20 \mathrm{fA} / \sqrt{\mathrm{Hz}}$. This small electronic noise level is also important because it allows a measurement of a false additive electronic noise asymmetry to the level of $0.5 \times 10^{-8}$ in a few minutes when beam is off.

Systematic errors are the big concern of the experiment. All known systematic error sources have been analyzed carefully ${ }^{23}$. The goal has been to design, build, and operate the experiment so that the known sources of systematic errors are suppressed at least 10 times smaller than the statistical error of $0.5 \times 10^{-8}$.

\section{Summary}

The $\vec{n}+p \rightarrow d+\gamma$ experiment will unambiguously measure the long-range pion-nucleon weak coupling constant $H_{\pi}^{1}$, thus probing the neutral current component of the hadronic weak interactions. The result will address the question of how the long-range weak force between nucleons is communicated to the underlying elementary quark-weak boson couplings of the standard model.

With a $1 / 10$ th of the scale of the $\vec{n}+p \rightarrow d+\gamma$ experiment we have measured $P V$ asymmetries in neutron capture on $\mathrm{Cl}$, La, and $\mathrm{Cd}$ with uncertainties of $10^{-6} 24$. An extrapolation from these results indicates that the estimates for a final error of the $\vec{n}+p \rightarrow d+\gamma$ experiment are correct. The commissioning of the $\vec{n}+p \rightarrow d+\gamma$ experiment will start in 2003 .

\section{Acknowledgement}

The work was supported in part by the U.S. Department of Energy, Office of Energy Research, under contract W-7405-ENG-36, and the National Science Foundation under grant PHY-9602872 and the MRI program PHY0100348 .

\section{References}

1. D. Tadic, Rep. Prog. Phys. 43, 67 (1980).

2. E. G. Adelberger and W. C. Haxton, Ann. Rev. Nucl. Part. Sci. 35, 501 (1985).

3. B. Desplanques, J. F. Donoghue, and B. R. Holstein, Ann. Phys. 124, 449 (1980).

4. W. Haeberli and B. R. Holstein, in Symmetries and Fundamental Interactions in Nuclei, ed. W. C. Haxton and E. M. Henley (World Scientific, Singapore, 1995) p. 17.

5. G. E. Mitchell, J. D. Bowman, S. I. Penttilä, and E. I. Sharapov, Phys. Reports 354, 157 (2001). 
6. R. Balzer et al., Phys. Rev. C 30, 1409 (1984); J. M. Potter et al., Phys. Rev. Lett. 33, 1307 (1974); D. E. Nagle et al., High Energy Physics with Polarized Beams and Polarized Targets, ed. G. H. Thomas, AIP Conf. Proc. No. 51 (AIP, New York, 1978); W. D. Ramsay et al., Can. J. Phys. 55, 79 (1999).

7. J. F. Cavaignac, B. Vignon, R. Wilson, Phys. Lett. 67B, 148 (1977).

8. C. A. Barnes et al., Phys. Rev. Lett. 40, 840 (1978); P. G. Bizetti et al., Lett. Nuovo Cimento 29, 167 (1982); G. Ahrens et al., Nucl. Phys. A390, 496 1982); S. A. Page et al., Phys. Rev. C 35, 1119 (1987); A. R. Berdoz et al., Phys. Rev. Lett. 87, 272301 (2001); M. Bini et al., Phys. Rev. Lett. 55, 795 (1985).

9. C. S. Wood et al., Science 275, 1759 (1997).

10. W. C. Haxton, Science 275, 1753 (1997); V. V. Flambaum and D. W. Murray, Phys. Rev. C 56, 1641 (1997); W. C. Haxton and C. E. Wieman, Annu. Rev. Nucl. Part. Sci. 51, 261 (2001); V. F. Dmitriev, Phys. Atom. Nucl. 64, 448 (2001).

11. W. C. Haxton, C.-P. Liu, and M. J. Ramsey-Musolf, Phys. Rev. Lett. 86, 5247 (2001).

12. W. S. Wilburn and J. D. Bowman, Phys. Rev. C 57, 3425 (1998).

13. T. Tomosovic et al., in these Proceedings.

14. P. Vetter et al., Phys. Rev. Lett. 74, 2658 (1995); N. H. Edwards et al., Phys. Rev. Lett. 74, 2654 (1995).

15. D. B. Kablan and M. S. Savage, Nucl. Phys. A556, 653 (1993); E. M. Henley, W.-Y. P. Hwang, and L. S. Kisslinger, Phys. Lett. B 367, 21 (1996); U.-G. Meissner and H. Weigel, Phys. Lett. B 447, 1 (1991); B. Desplanques, Phys. Lett. B 512, 305 (2001); C. H. Hyun, T.-S. Park, and D.-P. Min, Phys. Lett. B 516, 321 (2001); G. A. Lobov, Phys. Atom. Nucl. 65, 534 (2002).

16. P. D. Ferguson et al., in Proc. of ICANS-XIII (1995), PSI Report 95-02.

17. G. L. Jones et al., Nucl. Instr. and Meth. A 440, 772 (2000).

18. D. R. Rich et al., Nucl. Instr. and Meth. A 481, 431 (2002).

19. T. B. Smith et al., Nucl. Instr. and Meth. A, to be submitted (2002).

20. D. Renker, in Proc. of the ECFA Study Week on Instrumentation for HighLuminosity Hadron Collider (CERN, Geneva, 1989), Vol. CERN 89-10.

21. Hamamatsu, vacuum photo diode R2046PT.

22. A. Csótó, B. F. Gibson, G. L. Payne, Phys. Rev. C 56, 631 (1997).

23. The NPDGamma Proposal "Measurement of the Parity-Violating Gamma Asymmetry $A_{\gamma}$ in the Capture of Polarized Cold Neutrons by Para-Hydrogen", J. David Bowman (Spokesperson), LA-UR-1999-5432; W. M. Snow et al., Fundamental Physics with Pulsed Neutron Beams, ed. C. R. Could, G. L. Greene, and W. M. Snow, (World Scientific, Singapore, 2001) p. 203; W. M. Snow et al., Nucl. Instr. and Meth. A 440, 729 (2000).

24. G. S. Mitchell et al., Nucl. Instr. and Meth. A, submitted (2002). 